\title{
Scolia
}

Revue de linguistique

30 | 2016

Des connecteurs argumentatifs aux opérateurs discursifs

\section{(Et) dire que, connecteur concessif}

\section{Sandrine Deloor}

\section{(2) OpenEdition \\ Journals}

Édition électronique

URL : http://journals.openedition.org/scolia/477

DOI : $10.4000 /$ scolia.477

ISSN : 2677-4224

Éditeur

Presses universitaires de Strasbourg

\section{Édition imprimée}

Date de publication : 19 juin 2016

Pagination : 49-65

ISBN : 978-2-86820-944-3

ISSN : 1253-9708

Référence électronique

Sandrine Deloor, "(Et) dire que, connecteur concessif », Scolia [En ligne], 30 | 2016, mis en ligne le 09 avril 2019, consulté le 24 mai 2019. URL : http://journals.openedition.org/scolia/477 ; DOI : 10.4000/ scolia.477 
SCOLIA 30/2016, p. 48-66.

\title{
(Et) dire que, connecteur concessif
}

\author{
Sandrine DELOOR* \\ Université de Cergy-Pontoise, \\ UMR 7187 LDI \\ sandrinedeloor@yahoo.fr
}

\section{Introduction}

L'objectif de cet article est de construire un modèle permettant de prévoir l'interprétation des enchaînements dans lesquels figure le connecteur (Et) dire que. Par exemple:

1) Elle recula et réintégra le rang. "Hélas, voici la confirmation de mes craintes; elle est complètement folle», jugea MDA 802.

"Et dire que nous comptions sur elle pour nous sauver!» pensa le reste de l'unité. (Nothomb, Acide sulfurique, 2005)

2) Se frayant son chemin dans la foule, Arnaud Fécamp marchait vite. Tant bien que mal, Lucie essayait de le suivre, d'abord dans le sens de la masse, puis en luttant contre le flux une fois le stade dépassé. Bouches hurlantes, haleines chargées d'alcool, yeux rougis d'excitation. Dire que le match n'avait même pas commencé. (Thilliez, Gataca, 2011)

* Je remercie Jean-Claude Anscombre de m'avoir aidée à éviter plusieurs erreurs fatales dans cette étude et de m'avoir remise sur des pistes que j'avais abandonnées bien trop tôt. Bien entendu, sa responsabilité n'est pas pour autant engagée (cf. ex. 7a). 
3) Le système de chauffage n'avait jamais été le point fort de cet appartement qu'elle partageait depuis trois ans avec Colleen, sa colocataire.

Et dire que nous payons deux mille dollars de loyer! Soupira-t-elle. (Musso, Sauve-moi, 2005)

Après avoir examiné les définitions proposées par les dictionnaires et recensé les problèmes qu'elles soulèvent, nous présenterons une modélisation sémantique permettant (i) de calculer le sens de la relation concessive entre les entités sémantiques reliées par (Et) dire que, (ii) d'expliquer la structure de surface prototypique des enchaînements dans lesquels apparaît le connecteur et (iii) de prévoir l'interprétation des enchaînements dont la structure de surface n'est pas prototypique.

\section{Problématique}

Pour le Nouveau Petit Robert (1993: 650), (Et) dire que «exprime l'étonnement, l'indignation, la surprise». Pour Grevisse \& Goosse (2008: 507), «la tristesse, le regret, l'étonnement». Un examen de notre corpus (une centaine d'occurrences attestées, provenant de romans contemporains, de journaux et de recherches sur Internet) nous conduit à mettre en question ces définitions: si la tristesse, le regret et l'indignation sont souvent présents dans les exemples comportant (Et) dire que, force est de constater qu'ils n'apparaissent pas dans tous les énoncés de notre corpus. En (4), par exemple, le locuteur n'exprime aucun de ces trois sentiments, bien au contraire:

4) - Premier prix de la tombola, sourit Stern. On peut dire que vous êtes d'un naturel plutôt chanceux.

- Oui... Et dire que jai failli ne pas acheter de ticket. (Dicker, La vérité sur l'affaire Henry Quebert, 2012)

Seule la notion d'étonnement (dans un sens très large, ainsi que nous le verrons ci-dessous) semble pouvoir s'appliquer à l'ensemble des énoncés comportant $(E t)$ dire que. On remarque d'ailleurs que tous les énoncés de notre corpus admettent les enchaînements suivants: C'est fou quand on y pense, C'est incroyable quand même, Je n'en reviens pas, Comment est-ce possible?

Sans plus de précisions, la notion d'étonnement ne peut cependant être une notion opératoire dans le cadre d'une analyse sémantique. Un mot de la langue n'est pas une notion scientifique et ne permet 
pas de proposer des analyses reproductibles: comment distinguer de façon indiscutable ce qui est "étonnant» de ce qui ne l'est pas?, comment déjouer les pièges de la polysémie du mot étonnant en langue ?' Les deux exemples suivants nous permettront d'illustrer ces questionnements tout en poursuivant l'analyse:

- Si (Et) dire que marque un "étonnement", on peut se demander ce qui, dans un enchaînement de type $A$. (Et) dire que B., est présenté comme «étonnant»: est-ce A ? B ? ou bien encore la relation entre A et B? Pour le dictionnaire Antidote, "Le fait suivant est incroyable, surprenant par rapport au fait énoncé précédemment. Il a battu ce champion national d'échecs. (Et) dire qu'il n'a que douze ans!». Autrement dit, pour le dictionnaire Antidote, c'est B que le connecteur (Et) dire que présente comme étonnant par rapport à $\mathrm{A}$. Il nous semble que, dans l'exemple proposé, l'inverse est tout aussi vrai: on peut certes considérer que le fait qu'il n'ait que douze ans (B) est étonnant par rapport au fait qu'il ait battu un champion (A) mais on peut également considérer que le fait qu'il ait battu un champion (A) est étonnant par rapport au fait qu'il n'ait que douze ans (B). Sur la base de quels critères peut-on trancher entre ces deux analyses?

- On peut par ailleurs se demander si la notion d'étonnement est adaptée pour rendre compte de l'exemple (5):

5) Il s'appelle Félix Blanché, 53 ans. Il habite une maison à cinq cents mètres d'ici, où il vivait avec sa femme et son chien. Des policiers du coin et le médecin de famille sont chez lui. La femme est mal en point.

Le lieutenant Sharko imaginait aisément l'ampleur du choc. La pauvre épouse ne sén remettrait sans doute jamais. Dire qu'ils allaient devoir linterroger... (Thilliez, Pandemia, 2015)

Policier chevronné, le lieutenant Sharko ne pourrait pas se dire «étonné» de devoir interroger la femme d'un homme qui vient d'être tué: interroger les proches des victimes de meurtre est une procédure habituelle qu'il a de toute évidence suivie de nombreuses fois au cours de sa carrière. Le désespoir de la veuve n'a rien d' «étonnant» non plus: la perte d'un être cher est une épreuve douloureuse, à plus forte raison lorsqu'elle survient dans des circonstances aussi tragiques. Contrairement à ce qui a été observé dans l'exemple proposé par le

1 Sur ces questions, voir Deloor (2012: 45-49). 
dictionnaire Antidote, il n'y a rien «d'inattendu» ni «d'imprévu» dans la coexistence de A [La femme de la victime est effondrée] et B [Les policiers vont devoir l'interroger]. Si l'adjectif étonnant pouvait être appliqué à cet exemple, ce ne serait pas au sens où la coexistence de $\mathrm{A}$ et $\mathrm{B}$ "surprend par son caractère inattendu ou extraordinaire» (TLFI) mais au sens où elle "confond, frappe l'esprit par son étrangeté» (Nouveau Petit Robert: 833). L'adjectif étonnant serait ici plus proche de son sens étymologique («frapper de stupeur») que de son sens moderne. Sharko n'est pas «surpris» de devoir interroger une femme qui vient de perdre son mari, il est «stupéfait», "sidéré», "accablé» de vivre dans un monde où les policiers ne peuvent pas respecter le deuil des proches de victimes de meurtre. La violence de ce type de situation - qu'il a déjà vécue et qu'il vivra de nouveau - dépasse l'entendement, elle est «incroyable».

On le voit, il vaut mieux que nous abandonnions dès à présent l'idée de recourir aux adjectifs étonnant et incroyable pour décrire le connecteur (Et) dire que si nous ne voulons pas être obligée d'intituler le présent article «Des différentes manières d'accommoder les adjectifs étonnant, incroyable et tous leurs synonymes dans toutes leurs acceptions aux énoncés comportant le connecteur (Et) dire que»: la polysémie que nous venons d'évoquer est celle des adjectifs étonnant et incroyable et ce serait se tromper d'objet d'étude que de l'attribuer à (Et) dire que.

Le TLFI nous offre une porte de sortie:

DIRE

[...] 5. Loc. ou expr. formées avec l'inf. dire

[...] e) Avec que. [Avec une valeur concessive] (Et) dire que:

31. Dire qu'il était si joyeux, le jour où il avait quitté le service, après la guerre d'Italie, à l'idée de n'être plus un traîneur de sabre, un tueur de monde! (ZOLA, La Terre, 1887, p. 500)

Dans ce qui suit, nous nous appuierons sur la définition de la concession proposée par König (2006):

In uttering a complex sentence with a concessive clause, i.e., a sentence of the type Even though p, q (e.g., Even though it is raining, Fred is going out for a walk), a speaker is committed to the truth of both clauses $\mathrm{p}$ (It is raining) and $\mathrm{q}$ (Fred is going out for a walk) and asserts these two propositions against the background of an assumption that the two types of situations, $p$ and $q$, are generally incompatible. This background assumption or presupposition can 
roughly be described as follows: if $\mathrm{p}$, then normally not-q (If it is raining, one normally does not go out for a walk or The more it rains, the less people go out for a walk) [...]. How it is to be spelled out precisely is still a puzzle. What is clear, however, is that the situation described by a sentence with a concessive clause is an exception to a general tendency and therefore remarkable. (König, 2006: 820-821)

La définition proposée par le TLFI appelle plusieurs remarques:

- Tout d'abord, on remarquera que la notion de concession est en cohérence avec l'idée d'étonnement que nous venons d'examiner: si deux situations $\mathrm{A}$ et $\mathrm{B}$ sont perçues comme généralement incompatibles, alors la coexistence de ces deux situations a de fortes chances - même si ce n'est pas nécessairement le cas - d’être considérée comme «étonnante».

- La notion de concession suppose la mise en relation de deux situations. Pourtant, une seule situation est évoquée dans l'exemple proposé par le TLFI: où est la relation concessive dans Dire qu'il était si joyeux, le jour où il avait quitté le service, après la guerre d'Italie, à l'idée de n'être plus un traîneur de sabre, un tueur de monde!? Nous reproduisons ci-dessous le passage dont est tiré l'énoncé:

6) La souffrance, le dégoût des dix années passées à Rognes, lui remontaient de la poitrine en un flot de colère. Dire qu'il était si joyeux, le jour où il avait quitté le service, après la guerre d'Italie, à l'idée de n'être plus un traîneur de sabre, un tueur de monde! Et, depuis cette époque, il vivait dans de sales histoires, au milieu de sauvages. Dès son mariage, il en avait eu gros sur le cour; mais les voilà qui volaient, qui assassinaient, maintenant! De vrais loups, lâchés au travers de la plaine, si grande, si calme! (Zola, La Terre, 1887)

Dans cet extrait sont mis en perspective les espoirs que nourrissait Jean Macquart au moment où il a quitté l'armée (A [il pensait vivre une vie tranquille loin des horreurs de la guerre]) et la situation qu'il vit actuellement (B [il vit de sales histoires, au milieu de sauvages]). On remarquera que, dans cet exemple, les deux situations mises en contraste ne sont pas exposées de part et d'autre de la locution (Et) dire que. La configuration n'est pas $A$. (Et) dire que B. mais Dire que 
A. Et B. ${ }^{2}$ Doit-on en conclure que, contrairement à la terminologie que nous utilisons depuis le début de cette étude, (Et) dire que n'est pas un «connecteur»? Nous nous pencherons sur cette question en 2.3.

- On remarquera pour finir que l'incompatibilité entre A et B évoquée par les énoncés comportant $(E t)$ dire que peut prendre des aspects très divers:

\begin{tabular}{|r|l|l|}
\hline (1) & $\begin{array}{l}\text { A: [Elle est folle] } \\
\text { B: [Nous comptions sur elle pour nous sauver] }\end{array}$ & A aurait dû entraîner non B, ce n'est pas le cas. \\
\hline (5) & $\begin{array}{l}\text { A: [La femme de la victime est effondrée] } \\
\text { B: [Les policiers vont devoir l'interroger] }\end{array}$ & A devrait entraîner non B, ce n'est pas le cas. \\
\hline Antidote & $\begin{array}{l}\text { A: [ll a battu ce champion national d'échecs] } \\
\text { B: [ll a douze ans] }\end{array}$ & B aurait dû entraîner non A, ce n'est pas le cas. \\
\hline (2) & $\begin{array}{l}\text { A: [Les spectateurs sont ivres] } \\
\text { B: [Le match n'a pas commencé] }\end{array}$ & B devrait entrainer non A, ce n'est pas le cas. \\
\hline (3) & $\begin{array}{l}\text { A: [Le chauffage ne fonctionne pas] } \\
\text { B: [Nous payons deux mille dollars de loyer] }\end{array}$ & $\begin{array}{l}\text { A devrait entrainer non B, ce n'est pas le cas. } \\
\text { B devrait entraîner non A, ce n'est pas le cas. }\end{array}$ \\
\hline
\end{tabular}

L'un des objectifs de la modélisation sémantique que nous proposerons sera de prévoir ces variations.

\section{Modélisation sémantique}

\subsection{Sens de la relation concessive}

Comme nous venons de le voir, un enchaînement de type $A$. (Et) dire que $B$ peut indiquer une exception par rapport au principe général $<$ Si $\mathrm{A}$ alors non $\mathrm{B}>$ ou une exception par rapport au principe général $<$ Si B alors non $\mathrm{A}>$. Autrement dit, la structure de surface des énoncés comportant (Et) dire que ne permet pas de prévoir le sens de la relation concessive entre A et B.

Notre hypothèse est que ce n'est pas le connecteur (Et) dire que qui construit la relation concessive mais qu'il exploite une relation concessive préexistante.

Alors que, d'un point de vue logique, il y a équivalence entre la formule $p \rightarrow q$ et sa contraposée $\neg q \rightarrow \neg p$, ce n'est pas forcément le cas entre les principes généraux $\langle\mathrm{Si} \mathrm{p}$ alors $\mathrm{q}\rangle$ et $\langle\mathrm{Si}$ non $\mathrm{q}$ alors non $\mathrm{p}\rangle$

2 Remarquons que l'enchaînement serait malheureux sans la conjonction et devant depuis cette époque. 
en langue. Pour le montrer en évitant les problèmes d'interprétation des énoncés en $s i$ (en raison notamment des phénomènes d'abduction), nous poserons qu'une exception par rapport au principe général $<\mathrm{Si} \mathrm{p}$ alors $\mathrm{q}>$ peut être exprimée, en langue, au moyen des énoncés non $q$ alors que $p$ et $q$ devrait être le cas étant donné que $p$ est le cas. Sur cette base, il est aisé de constater que les principes généraux $<\mathrm{Si} p$ alors $\mathrm{q}>$ et $<$ Si non $\mathrm{q}$ alors non $\mathrm{p}>$ ne sont pas forcément équivalents en langue:

\begin{tabular}{|c|c|}
\hline $\begin{array}{l}\text { Exception au principe général }<\text { Si } p \text { alors } q>\text { : } \\
\text { non q alors que } p \\
q \text { devrait être le cas étant donné que p est le cas }\end{array}$ & $\begin{array}{l}\text { Exception au principe général <Si [pleuvoir] alors [ne } \\
\text { pas sortir]>: } \\
\text { (a) Je sors alors qu'il pleut. } \\
\text { (b) Je ne devrais pas sortir étant donné qu'il pleut. }\end{array}$ \\
\hline $\begin{array}{l}\text { Exception au principe général }<\text { Si non q alors non } p>\text { : } \\
\text { palors que non q } \\
\text { p ne devrait pas être le cas étant donné que q n'est pas } \\
\text { le cas }\end{array}$ & $\begin{array}{l}\text { Exception au principe général <Si [sortir] alors [ne pas } \\
\text { pleuvoir]>: } \\
\text { (c) *Il pleut alors que je sors. } \\
\text { (d) *Il ne devrait pas pleuvoir étant donné que je sors. }\end{array}$ \\
\hline
\end{tabular}

L'inacceptabilité de (c) et (d) montre que le principe général $<$ Si [sortir] alors [ne pas pleuvoir] $>$ n'est pas valable en langue, contrairement au principe général $<\mathrm{Si}$ [pleuvoir] alors [ne pas sortir] $>$ correspondant au stéréotype ${ }^{3}$ Généralement, les gens ne sortent pas quand il pleut.

Appliquées aux énoncés reliés par (Et) dire que dans les exemples de la section précédente, les paraphrases proposées permettent de calculer le sens de la relation concessive existant entre eux. Précisons que, lorsque $\mathrm{P}$ est au passé, la paraphrase obtenue n'est pas $P$ ne devrait pas être le cas étant donné que $Q$ est le cas mais $P$ n'aurait pas dî être le cas étant donné que $Q$ est le cas:

3 Sur la théorie des stéréotypes, voir notamment Anscombre (2010). 
Cas 1: A ne devrait pas / n'aurait pas dû être le cas étant donné que B est le cas

\begin{tabular}{|c|c|c|}
\hline & \begin{tabular}{l}
\multicolumn{1}{c}{ Exemple 2} \\
A: [Les spectateurs sont ivres] \\
B: [Le match n'a pas commencé]
\end{tabular} & $\begin{array}{l}\text { Exemple d'Antidote } \\
\text { A: [ll a battu ce champion national } \\
\text { d'échecs] } \\
\text { B: [ll a douze ans }]\end{array}$ \\
\hline $\begin{array}{l}\text { Il est possible de dire: } \\
\text { A alors que } B \text {. }\end{array}$ & $\begin{array}{l}\text { Les spectateurs sont ivres alors que le } \\
\text { match n'a pas commencé. }\end{array}$ & $\begin{array}{l}\text { Il a battu ce champion national d'échecs alors } \\
\text { qu'il a douze ans. }\end{array}$ \\
\hline $\begin{array}{l}\text { A ne devrait pas / n'aurait pas } \\
\text { dû être le cas étant donné que } \\
\text { Best le cas. }\end{array}$ & $\begin{array}{l}\text { Les spectateurs ne devraient pas être } \\
\text { ivres étant donné que le match n'a } \\
\text { pas commencé. }\end{array}$ & $\begin{array}{l}\text { Il n'aurait pas dû battre ce champion natio- } \\
\text { nal d'échecs étant donné qu'il a douze ans. }\end{array}$ \\
\hline $\begin{array}{l}\text { Et non: } \\
\text { B alors que } A \text {. }\end{array}$ & $\begin{array}{l}\text { *Le match n'a pas commencé alors } \\
\text { que les spectateurs sont ivres. }\end{array}$ & $\begin{array}{l}\text { *Il a douze ans alors qu'il a battu ce cham- } \\
\text { pion national d'échecs. }\end{array}$ \\
\hline $\begin{array}{l}\text { B ne devrait pas / n'aurait pas } \\
\text { dû être le cas étant donné que } \\
\text { A est le cas. }\end{array}$ & $\begin{array}{l}\text { *Le match devrait avoir commencé } \\
\text { étant donné que les spectateurs sont } \\
\text { ivres. }\end{array}$ & $\begin{array}{l}\text { *ll ne devrait pas avoir douze ans étant } \\
\text { donné qu'il a battu ce champion national } \\
\text { d'échecs. }\end{array}$ \\
\hline $\begin{array}{l}\text { Donc on a affaire à une ex- } \\
\text { ception au principe général } \\
<\text { Si B alors non } A>\end{array}$ & $\begin{array}{l}\text { Si [le match ne pas avoir com- } \\
\text { mencé] alors [les spectateurs ne pas } \\
\text { être ivres]> }\end{array}$ & $\begin{array}{l}\text { <Si [avoir douze ans] alors [ne pas battre ce } \\
\text { champion national d'échecs] }>\end{array}$ \\
\hline $\begin{array}{l}\text { Et non au principe général } \\
<\text { Si } A \text { alors non } B>\end{array}$ & $\begin{array}{l}\text { Et non < Si [les spectateurs être } \\
\text { ivres] alors [le match avoir com- } \\
\text { mencé }]>\end{array}$ & $\begin{array}{l}\text { Et non }<\text { Si [battre ce champion national } \\
\text { d'échecs }>\text { alors [ne pas avoir douze ans] }>\end{array}$ \\
\hline Stéréotype & $\begin{array}{l}\text { Généralement, les spectateurs ne } \\
\text { sont pas ivres quand le match n'a pas } \\
\text { commencé. }\end{array}$ & $\begin{array}{l}\text { Généralement, les enfants de douze ans ne } \\
\text { battent pas les champions nationaux dans } \\
\text { leur discipline. }\end{array}$ \\
\hline
\end{tabular}


Cas 2: B ne devrait pas / n'aurait pas dû être le cas étant donné que A est le cas

\begin{tabular}{|c|c|c|}
\hline & $\begin{array}{l}\quad \text { Exemple } 5 \\
\text { A: [La femme de la victime est effon- } \\
\text { drée] } \\
\text { B: [Les policiers vont devoir l'interroger] }\end{array}$ & $\begin{array}{l}\quad \text { Exemple } 1 \\
\text { A: [Elle est folle] } \\
\text { B: [Nous comptions sur elle pour nous } \\
\text { sauver] }\end{array}$ \\
\hline $\begin{array}{l}\text { Il est possible de dire: } \\
\text { B alors que } A \text {. }\end{array}$ & $\begin{array}{l}\text { Les policiers interrogent la femme de la } \\
\text { victime alors qu'elle est effondrée. }\end{array}$ & $\begin{array}{l}\text { Nous comptions sur elle pour nous sau- } \\
\text { ver alors qu'elle est folle. }\end{array}$ \\
\hline $\begin{array}{l}\text { B ne devrait pas / n'aurait pas } \\
\text { dû être le cas étant donnéque } \\
\text { A est le cas. }\end{array}$ & $\begin{array}{l}\text { Les policiers ne devraient pas interroger la } \\
\text { femme de la victime étant donné qu'elle } \\
\text { est effondrée. }\end{array}$ & $\begin{array}{l}\text { Nous n'aurions pas dû compter sur elle } \\
\text { pour nous sauver étant donné qu'elle } \\
\text { estfolle. }\end{array}$ \\
\hline $\begin{array}{l}\text { Et non: } \\
\text { A alors que } B \text {. }\end{array}$ & $\begin{array}{l}\text { * La femme de la victime est effondrée } \\
\text { alors que les policiers l'interrogent. }\end{array}$ & $\begin{array}{l}\text { *Elle est folle alors que nous comptions } \\
\text { sur elle pour nous sauver. }\end{array}$ \\
\hline $\begin{array}{l}\text { A ne devrait pas / n'aurait pas } \\
\text { dû être le cas étant donnéque } \\
\text { Best le cas. }\end{array}$ & $\begin{array}{l}\text { * La femme de la victime ne devrait pas } \\
\text { être effondrée étant donné que les poli- } \\
\text { ciers l'interrogent. }\end{array}$ & $\begin{array}{l}\text { *Elle ne devrait pas être folle étant } \\
\text { donné que nous comptions sur elle pour } \\
\text { nous sauver. }\end{array}$ \\
\hline $\begin{array}{l}\text { Donc on a affaire à une ex- } \\
\text { ception au principe général } \\
<\text { Si } A \text { alors non } B>\end{array}$ & $\begin{array}{l}<\text { Si [la femme de la victime être effon- } \\
\text { drée] alors [les policiers ne pas l'inter- } \\
\text { roger]> }\end{array}$ & $\begin{array}{l}<\text { Si [elle être folle] alors [nous ne pas } \\
\text { compter sur elle] }>\end{array}$ \\
\hline $\begin{array}{l}\text { Et non au principe général } \\
\langle\text { Si B alors non } A>\end{array}$ & $\begin{array}{l}\text { Et non <Si [les policiers l'interroger] } \\
\text { alors [la femme de la victime ne pas être } \\
\text { effondrée] }>\end{array}$ & $\begin{array}{l}\text { Et non <Si [nous compter sur elle] alors } \\
\text { [elle ne pas être folle] }>\end{array}$ \\
\hline Stéréotype & $\begin{array}{l}\text { On doit laisser les personnes endeuillées } \\
\text { en paix. }\end{array}$ & On ne doit pas compter sur les fous. \\
\hline
\end{tabular}


Cas 3 : contraposition possible $>$ double interprétation

\begin{tabular}{|c|c|}
\hline \multicolumn{2}{|c|}{ Exemple 3} \\
\hline $\begin{array}{l}\| \text { est possible de dire: } \\
B \text { alors que } A \text {. }\end{array}$ & $\begin{array}{l}\text { Nous payons deux mille dollars de loyer alors que le } \\
\text { chauffage ne fonctionne pas. }\end{array}$ \\
\hline Bne devrait pas être le cas étant donné que A est le cas. & $\begin{array}{l}\text { Nous ne devrions pas payer deux mille dollars de loyer } \\
\text { étant donné que le chauffage ne fonctionne pas. }\end{array}$ \\
\hline $\begin{array}{l}\text { Et: } \\
\text { A alors que } B \text {. }\end{array}$ & $\begin{array}{l}\text { Le chauffage ne fonctionne pas alors que nous payons } \\
\text { deux mille dollars de loyer. }\end{array}$ \\
\hline A ne devrait pas être le cas étant donné que B est le cas. & $\begin{array}{l}\text { Le chauffage devrait4 fonctionner étant donné que nous } \\
\text { payons deux mille dollars de loyer. }\end{array}$ \\
\hline $\begin{array}{l}\text { Donc on a affaire à une exception au principe général } \\
<\text { Si A alors non } B>\end{array}$ & $\begin{array}{l}<\text { Si [le chauffage ne pas fonctionner> alors [nous ne } \\
\text { pas payer deux mille dollars de loyer] }\end{array}$ \\
\hline ou au principe général $<i B$ alors non $A>$ & $\begin{array}{l}\text { ou }<\text { Si [nous payer deux mille dollars de loyer] alors [le } \\
\text { chauffage fonctionner] }\end{array}$ \\
\hline Stéréotype & $\begin{array}{l}\text { Généralement, quand on paye un loyer élevé, les équipe- } \\
\text { ments de base fonctionnent. } \\
\text { Généralement, quand les équipements de base ne fonc- } \\
\text { tionnent pas, on ne paye pas un loyer élevé. }\end{array}$ \\
\hline
\end{tabular}

\subsection{Un connecteur porte-manteau?}

Dans la mesure où la structure de surface des énoncés comportant (Et) dire que ne permet pas de prévoir le sens de la relation concessive entre $\mathrm{A}$ et $\mathrm{B}$, on pourrait penser que (Et) dire que a la propriété de marqueur "porte-manteau", à l'instar de pourtant et cependant:

Pourtant et cependant se différencient des autres marqueurs par plusieurs propriétés: ils peuvent relier deux propositions qui sont permutables entre elles, sans que le sens de la relation concessive entre les deux propositions soit modifié. R.-L. Wagner qualifiait les marqueurs ayant cette propriété de marqueurs «porte-manteau». La valeur de la relation marquée par ces deux adverbes est celle de la concession logique:

4 Notons que le verbe devoir a une valeur déontique dans certaines paraphrases et une valeur aléthique dans d'autres. Cette variation ne nous paraît pas remettre en cause le caractère systématique des relations observées: dans toutes les paraphrases, le verbe devoir caractérise la situation qui serait «normale», «attendue» au vu d'un principe général donné. 
Notre voiture a cent mille bornes, elle a pourtant (cependant) très bien roulé.

Bien que notre voiture ait cent mille bornes, elle a très bien roulé.

Notre voiture a très bien roulé, elle a pourtant (cependant) cent mille bornes.

Notre voiture a très bien roulé, bien qu'elle ait cent mille bornes.

(Morel, 1996: 53)

On remarque cependant que la permutation de $\mathrm{A}$ et $\mathrm{B}$ peut donner lieu à des enchaînements inacceptables dans des cas où les tests présentés en 2.1. semblent indiquer que la contraposition est possible (cas 3):

7) a. Mon article est nul. Et dire qu'on m'a aidé!

b. ${ }^{*} O n$ m'a aidé. Et dire que mon article est nul!

\begin{tabular}{|c|c|}
\hline \multicolumn{2}{|l|}{$\begin{array}{l}\text { A : [Mon article est nt } \\
\text { B: [0n m'a aidé] }\end{array}$} \\
\hline $\begin{array}{l}\text { Il est possible de dire: } \\
\text { B alors que } A \text {. } \\
\text { Bn'aurait pas dû être le cas étant donnéque A est le cas. }\end{array}$ & $\begin{array}{l}\text { On m'a aidé alors que mon article est nul. } \\
\text { On n'aurait pas dû m'aider étant donné que mon article } \\
\text { est nul. }\end{array}$ \\
\hline $\begin{array}{l}\text { Et: } \\
\text { A alors que B. } \\
\text { A n'aurait pas dû être le cas étant donné que B est le cas. }\end{array}$ & $\begin{array}{l}\text { Mon article est nul alors qu'on m'a aidé. } \\
\text { Mon article n'aurait pas dû être nul étant donnéqu'on m'a } \\
\text { aidé. }\end{array}$ \\
\hline $\begin{array}{l}\text { Donc on a affaire à une exception au principe général } \\
<\text { Si } A \text { alors non } B> \\
\text { ou au principe général }\langle\text { Si } B \text { alors non } A>\end{array}$ & $\begin{array}{l}<\text { Si [mon article être nul] alors [on ne pas m'avoir aidé] }> \\
\text { ou <Si [on m'avoir aidé] alors [mon article ne pas être } \\
\text { nul] }>\end{array}$ \\
\hline Stéréotype & $\begin{array}{l}\text { Généralement, quand quelqu'un a écrit un article nul, on } \\
\text { nel'aide pas. } \\
\text { Généralement, quand on est aidé pour écrire un article, } \\
\text { l'article n'est pas nul. }\end{array}$ \\
\hline
\end{tabular}

Il est intéressant d'observer que, contrairement à ce qui se passait dans l'exemple (3), il n'y a pas d'équivalence ici entre les deux paires de paraphrases produites par l'application des tests proposés. Dans la première ( $B$ alors que $A$ ), le locuteur ne parle pas d'une aide qu'il a reçue pour rédiger son article mais d'une aide qu'il a reçue après la rédaction de son article, malgré la nullité de son article. Dans la seconde, en revanche ( $A$ alors que $B$ ), c'est pour rédiger son article que le locuteur a reçu de l'aide et il se lamente que, malgré cette aide, son 
article soit nul. C'est à cette seconde interprétation et en aucun cas à la première que correspond l'exemple (7a). Comment l'expliquer?

Notre hypothèse est que, dans un énoncé de type $A$. (Et) dire que $B$, le segment (Et) dire que $B$ se présente comme un commentaire à propos de A et doit par conséquent être interprété dans le cadre de A. En (7a), (Et) dire que impose d'interpréter On m'a aidé par rapport à Mon article est nul. On m'a aidé est ainsi compris comme [On m’a aidé à écrire cet article]. Les rapports d'anaphore que l'on peut observer dans la plupart des enchaînements comportant (Et) dire que vont dans le sens de notre hypothèse, qu'il s'agisse d'anaphores associatives (exemples 2 et 3 ) ou d'anaphores pronominales (exemples 1 et 7 et exemple d'Antidote). En (2), par exemple, c'est au match auquel viennent assister les spectateurs déjà ivres dont il a été question dans le premier énoncé que renvoie le syntagme le match; en (1) il doit être interprété comme [celui qui a battu ce champion national d'échecs], etc.

Si cette hypothèse était suffisante pour expliquer l'inacceptabilité de (7b), l'explicitation de la relation entre le premier segment et le second devrait permettre de rendre l'enchaînement acceptable. Or force est de constater que (7c) est tout aussi bizarre que (7b):

7) c. ${ }^{*} O n$ mia aidé à écrire cet article. Et dire qu'il est nul!

$(E t)$ dire que n'est donc pas un marqueur "porte-manteau», même si la structure de surface des énoncés dans lesquels il apparaît n'est pas contrainte par le sens de la relation concessive entre A et B. Quelles sont les règles qui régissent cette structure de surface?

Notre hypothèse est que tout énoncé comportant (Et) dire que met en perspective deux situations: une situation $\mathrm{Y}$ saillante pour le locuteur au moment de l'énonciation (parce qu'elle vient de se produire ou parce que le locuteur vient de la (re)découvrir) et une situation $\mathrm{X}$ dont le locuteur avait connaissance avant l'énonciation. Ayant en tête $\mathrm{Y}$, le locuteur se remémore $\mathrm{X}$ et met en scène son trouble face à la coexistence des deux situations: l'une des deux ne devrait pas / n'aurait pas dû être le cas étant donné que l'autre est le cas. Dans les énoncés de type A. (Et) dire que B., la situation Y est exprimée par le segment $A$ tandis que la situation $X$ est exprimée par le segment $B$.

Bien qu'approximative, la glose «Placé devant $\mathrm{Y}$, je me remémore $\mathrm{X}$ » permet de traduire le caractère saillant de $\mathrm{Y}$ au moment de l'énonciation et l'antériorité de $\mathrm{X}$ par rapport à $\mathrm{Y}$. On peut ainsi 
expliquer l'inacceptabilité de (7c) et prédire la possibilité d'une permutation entre les segments A et B dans l'exemple (3):

Glose traduisant la relation entre $\mathrm{X}$ et $\mathrm{Y}$ en $(7 \mathrm{a})$ : «Placé devant le fait que mon article est nul, je me remémore qu'on m'a aidé à l'écrire» => Mon article est nul. Et dire qu'on m'a aidé!

Glose traduisant la relation entre $\mathrm{X}$ et $\mathrm{Y}$ en $(7 \mathrm{c})$ : « ${ }^{\star}$ Placé devant le fait qu'on m'a aidé à écrire cet article, je me remémore qu'il est nul.» $=>^{*} O n$ m'a aidé à écrire cet article. Et dire qu'il est nul!

Glose traduisant la relation entre X et Y en (3): "Placé devant le fait que le chauffage ne fonctionne pas, je me remémore que je paye 2000 dollars de loyer" $=>$ Le chauffage ne fonctionne pas. Et dire que je paye 2000 dollars de loyer!

Autre glose possible à partir de (3) : «Placé devant le fait que je paye 2000 dollars de loyer (je suis par exemple en train de remplir le chèque du loyer ou je viens d'apprendre que mon loyer a augmenté), je me remémore que le chauffage ne fonctionne pas» => Je paye 2000 dollars de loyer. Et dire que le chauffage ne fonctionne pas!

Selon notre hypothèse, c'est donc l'antériorité de $\mathrm{X}$ par rapport à Y qui explique l'impossibilité de permuter A et B dans la plupart des exemples comportant (Et) dire que. La dépendance de B par rapport à $\mathrm{A}$ et les relations d'anaphore observées précédemment sont une conséquence de ce trait sémantique.

\subsection{Configurations syntaxiques}

Soit l'énoncé suivant:

8) Et dire que la peine de mort a été abolie!

On conviendra que cet énoncé ne peut être interprété de façon isolée. Pour le comprendre, il est nécessaire de connaître la situation dans laquelle il a été émis. Par exemple:

Situation d'énonciation: Le locuteur regarde le journal télévisé. Il y est question du meurtre d'un suspect dans le cadre d'une intervention de police: d'après les premiers éléments de l'enquête interne, les policiers n'étaient pas menacés par le suspect et ils l'ont tué volontairement. 
Interprétation: $\mathrm{Y}$ : [L'Etat continue à exécuter des criminels]; $\mathrm{X}$ : [La peine de mort a été abolie]

Glose traduisant la relation entre $\mathrm{X}$ et $\mathrm{Y}$ : «Placé devant le fait que l'Etat continue à exécuter des criminels, je me remémore que la peine de mort a été abolie.»

Paraphrases traduisant la relation concessive sous-jacente: L'Etat continue à exécuter des criminels alors que la peine de mort a été abolie; L'Etat ne devrait pas continuer à exécuter des criminels étant donné que la peine de mort a été abolie.

Situation d'énonciation: Le locuteur regarde un reportage détaillant les crimes commis par un tueur en série.

Interprétation: $\mathrm{Y}$ : [Il y a toujours des crimes horribles] ; X: [La peine de mort a été abolie]

Glose traduisant la relation entre $\mathrm{X}$ et $\mathrm{Y}$ : «Placé devant le fait qu'il y a toujours des crimes horribles, je me remémore que la peine de mort a été abolie.»

Paraphrases traduisant la relation concessive sous-jacente: $L a$ peine de mort a été abolie alors qu'il y a toujours des crimes horribles.; La peine de mort n'aurait pas dû être abolie étant donné qu'il y a toujours des crimes horribles.

Il est intéressant de constater qu'une personne qui ne connaît pas le contenu du reportage que le locuteur est en train de regarder mais qui connaît la position de celui-ci par rapport à la peine de mort peut interpréter de façon partielle l'énoncé (8):

Interprétation: Y; X: [La peine de mort a été abolie]

Glose traduisant la relation entre $\mathrm{X}$ et $\mathrm{Y}$ : «Placé devant $\mathrm{Y}$, je me remémore que la peine de mort a été abolie.»

Paraphrases traduisant les relations concessives sous-jacentes possibles:

P1: Y alors que la peine de mort a été abolie; Y ne devrait pas être le cas étant donné que la peine de mort a été abolie.

P2: La peine de mort a été abolie alors que Y; La peine de mort n'aurait pas dî être abolie étant donné que $Y$.

\section{Reconstitution:}

[Le locuteur de (8) est contre la peine de mort] > Le locuteur est choqué par ce qu'il vient de voir car ça ne devrait pas 
être possible dans un pays où la peine de mort a été abolie (paraphrase P1)

[Le locuteur de (8) est pour la peine de mort] > Ce que vient de voir le locuteur l'a conforté dans son idée que la peine de mort n'aurait pas dû être abolie (paraphrase P2).

Cet exemple montre qu'il est nécessaire d'établir une distinction entre entités sémantiques et segments matériels: l'hypothèse que nous avons proposée repose sur l'idée que la locution (Et) dire que met en relation deux entités sémantiques, quelle que soit la configuration syntaxique dans laquelle elle apparait. C'est en ce sens que nous qualifions (Et) dire que de "connecteur» (cf. Anscombre \& Delahaie: 2014). Comme nous venons de le voir, la configuration $\varnothing$. (Et) dire que $B$. est parfaitement possible ${ }^{5}$ mais son interprétation impose de prendre en compte la situation de discours pour reconstituer Y.

L'exemple proposé par le TLFI illustre une autre configuration syntaxique fréquente: $\varnothing$. (Et) dire que $A M C B$ (où $M C$ est un marqueur concessif). Comme dans le cas précédent, le fait que cette configuration soit possible ne remet pas en cause le terme de "connecteur» utilisé tout au long de cette étude puisque, répétons-le, nous utilisons ce terme pour caractériser le fonctionnement sémantique de (Et) dire que et non la structure de surface des énoncés dans lesquels il apparaît. On trouvera deux autres exemples de cette configuration ci-dessous:

$\mathrm{MC}=$ alors que

9) Je n'ai jamais compris pourquoi il aimait tant me regarder manger, mais je m'exécutai avec joie. Dire que d'aucuns assassinent des poulpes alors qu'il y a des kakis mûrs à dévorer! (Nothomb, Ni d'Eve ni d'Adam, 2007)

$\mathrm{MC}=$ et de clash

10) Amandine se sentait mal. Dire qu'elle avait travaillé à proximité de Séverine, et qu'elle n'avait rien vu. (Thilliez, Pandemia, 2015)

5 C'était aussi celle de l'exemple (3). Notons que la configuration $A$. (Et) dire que Ø est en revanche impossible: ${ }^{*}$ Il a battu ce champion national d'échecs. Et dire que. 


\section{Conclusion}

Si la modélisation sémantique que nous venons de proposer permet de prévoir l'interprétation de la majorité des enchaînements comportant (Et) dire que, plusieurs questions restent en suspens.

- On remarque tout d'abord que les paraphrases $P$ alors que $Q$ et $P$ ne devrait pas être le cas étant donné que $Q$ ne sont pas satisfaisantes dans tous les cas. Par exemple, dans les énoncés où (Et) dire que introduit une opinion du locuteur (Y. (Et) dire que je pensais que E), la paraphrase $Y$ alors que je pensais que E est acceptable mais pas sa "dérivée» $Y$ ne devrait pas être le cas étant donné que je pensais que $E$. En fait, les exemples construits sur ce modèle ne signalent pas une exception à un principe $<$ [si moi penser que $\mathrm{E}]$ alors [non $\mathrm{Y}]>$ mais une situation contraire aux croyances du locuteur: le locuteur croit que $\mathrm{E}$; si E était vrai, alors $\mathrm{Y}$ ne serait pas le cas; $\mathrm{Y}$ étant le cas, on en déduit que E n'est pas vrai : le locuteur se trompait. On rencontre un problème similaire dans les énoncés de type $Y$. (Et) dire que E a failli être le cas (cf. exemple 4). De nouveau, la paraphrase $Y$ alors que E a failli être le cas est acceptable mais pas sa « dérivée» $Y$ ne devrait pas être le cas étant donné que E a failli être le cas. Dans ce type d'exemple, E implique non Y mais c'est non E qui s'est produit donc Y. On le voit, le modèle que nous proposons doit encore être affiné.

- Une autre question mériterait selon nous d'être approfondie. En 2.2., lorsque nous nous sommes servie de l'enchaînement (7c) ${ }^{\star} O n$ m'a aidé à écrire cet article. Et dire qu'il est nul! pour montrer l'antériorité de $\mathrm{X}$ par rapport à $\mathrm{Y}$, nous ne nous sommes pas penchée sur les spécificités de l'énoncé Il est nul. Or on se rend compte que (Et) dire que ne peut jamais introduire un énoncé évaluatif et ce, quelle que soit la situation Y. ${ }^{\star} E t$ dire qu'elle est gentille, ${ }^{\star} E t$ dire que c'est un bon film sont inacceptables sauf s'ils sont interprétés comme des citations (Et dire que tu la trouves gentille, Et dire que tout le monde trouve que c'est un bon film). On comparera ainsi ${ }^{\star} E t$ dire qu'elle est gentille et $E t$ dire qu'elle est gentille avec lui, où (Et) dire que redevient acceptable car l'énoncé n'est plus évaluatif mais factuel. Cette observation ne remet pas en cause notre hypothèse, bien au contraire: selon nous, c'est précisément parce qu'il y a une antériorité de $\mathrm{X}$ par rapport à $\mathrm{Y}$ que (Et) dire que ne peut introduire un énoncé évaluatif. Notre impression est que, dans la plupart des exemples où $\mathrm{X}$ est un état, $\mathrm{Y}$ est un événement 
incompatible avec cet état (dans ces exemples, le sens de la relation concessive est donctoujours $\langle\mathrm{Si} \mathrm{X}$, alors non $\mathrm{Y}\rangle$ ). $\mathrm{X}$ ne peut donc pas être une évaluation: comment le locuteur pourrait-il continuer à soutenir que quelqu'un est gentil ou qu'un film est bon s'il vient de découvrir un événement incompatible avec cette évaluation?

\section{Bibliographie}

ANSCOMBRE J.-C. \& DELAHAIE J. (2014), Connecteurs et connexion : quelques jalons, Cahiers de lexicologie 105, 161-179.

ANSCOMBRE J.-C. (2010), A la croisée des chemins: la théorie des stéréotypes, in Alvarez Castro C., Bango de la Campa F. \& Donaire M.L. (éds), Liens linguistiques, Berne, Peter Lang, 7-23. Antidote RX (2007), version 4 [Logiciel], Montréal, Druide informatique.

Trésor De La Langue Française Informatisé (TLFI): http://atilf.atilf.fr/ tlf.htm

DELOOR S. (2012), Les hypothèses observationnelles en sémantique: Qui ne glose rien n'a rien, Cuadernos de Filología Francesa 23, 37-53.

GREVISSE M. \& GOOSSE A. (2008), Le bon usage, Bruxelles, De Boeck-Duculot.

KÖNIG E. (2006), Concessive clauses, in Brown K. (éd.), Encyclopedia of Language \& Linguistics, Elsevier, 820-824.

MOREL M.-A. (1996), La concession en français, Paris, Ophrys.

REY-DEBOVE J. \& REY A. (éds) (1993), Le Nouveau Petit Robert, Paris, Dictionnaires Le Robert. 